\title{
Measuring Wellbeing in a Healthcare Setting: a Qualitative Study of Staff and Service User Perspectives
}

\author{
Sophie Attwood ${ }^{1}$ (D) Tarra L. Penney ${ }^{1} \cdot$ Rachael O'Leary $^{1} \cdot$ Charlotte Klahn $^{1}$. \\ Benjamin Kelly ${ }^{1}$
}

Received: 15 December 2017 / Accepted: 4 October 2018 / Published online: 25 October 2018

(C) The Author(s) 2018

\begin{abstract}
This study explored staff and service users' views on a selection of candidate wellbeing questionnaires for use by a UK-based healthcare provider, Nuffield Health, that is embarking on a programme to monitor the impact of their services on wellbeing. Ten stakeholder focus groups were conducted with 64 staff and service users, in addition to three in-depth telephone interviews with Nuffield Health Executive Board members. Focus groups and interviews were audio-recorded and transcripts examined using qualitative thematic analysis. Across focus groups, stakeholders commented on the importance of ensuring that data from wellbeing questionnaires provided actionable insight that can help to direct care and inform service improvements. Service users conceptualised their own wellbeing as a broad concept that encompassed more than just a mental state, and that was broadly aligned to the notion of feeling "in balance". Following the measurement process, service users were particularly interested to receive feedback on determinants of their own wellbeing, beyond those directly related to the services provided by Nuffield Health. Staff discussed the importance of embedding wellbeing measurement within a supportive service infrastructure and ensuring that appropriate training is available to help those with distinct wellbeing-related needs if identified through the monitoring process. Wellbeing is complex and subjective concept, and existing questionnaires used by Nuffield Health are inappropriate for measuring the independent impact of a health service on user wellbeing. Further work is required to pilot test alternative questionnaires that better reflect service users' intuitive understandings of their own wellbeing, and that can provide actionable data to inform ongoing service improvements.
\end{abstract}

Keywords Wellbeing · Health service · Qualitative research · Service evaluation · Wellbeing scale

Electronic supplementary material The online version of this article (https://doi.org/10.1007/s11482-0189664-6) contains supplementary material, which is available to authorized users.

Sophie Attwood

sophieattwood24@gmail.com

Extended author information available on the last page of the article 


\section{Introduction}

Use of the term "wellbeing" has grown in popularity recent years, with a plethora of tools and services now available to assist those who may wish to improve their wellbeing in doing so. These hold potential value for healthcare providers who are increasingly looking for novel and evidence-based services to assist in their fight against lifestyle-related illnesses, both physical and mental (Naylor et al. 2015; NHS 2014). To date, a range of services and techniques, including stress management, physical activity promotion, relaxation training and mindfulness meditation, have proven effective. This is both in terms of enhancing the wellbeing of patients currently undergoing care (Mikolasek et al. 2017), as well as aiding primary prevention efforts in healthy populations (Dooris et al. 2017; Enns et al. 2016; Murray et al. 2016).

In the United Kingdom (UK), for example, one service that has proved particularly successful in improving wellbeing in a healthcare setting is Improving Access to Psychological Therapy (IAPT) (UK National Health Service; NHS). This initiative offers short-term, evidence-based psychological support to individuals suffering from anxiety, depression and stress (NHS England 2018), while the NHS is also utilising alternatives such as "mindfulness" meditation to assist patient groups in managing the symptoms of a range of different illnesses, such as chronic pain, sleep problems or fatigue (Hilton et al. 2017). Private health care providers in the UK are similarly interested in helping patient groups to improve their wellbeing and have been able to implement a range of innovative "wellbeing" services, including online access to a wider number of professionals, not just General Practitioners (GPs) offering health assessments tailored to personal risk profiles, but also exercise and nutrition acting as first line preventative and treatment-related strategies (NH 2018).

In addition to developing and implementing new wellbeing-promoting services, the concept of "wellbeing" is also of interest to healthcare providers seeking ways to understand more about the impact of their services (Coulter 2017). Data from wellbeing questionnaires is seen as one useful way to compare the outcomes of different health services using a common metric, as well as to track improvements in patient wellbeing following interaction with a single service line over time. Furthermore, if the same wellbeing questionnaire is utilised by different providers and the resultant data made publically available, benchmarking between providers becomes possible. This has positive implications for facilitating patient choice when comparing between and selecting a health care provider (Benning et al. 2015; Ettorchi-Tardy et al. 2012; Lee et al. 2013).

A further proposed benefit of adopting wellbeing questionnaires to monitor the impact of health services is that these may yield data that providers can use to understand the broader impact of their services on patients' lives, beyond considering condition-specific outcome measures only. Moreover, data from wellbeing questionnaires can also allow comparisons between treatment-related services delivered in more traditional healthcare contexts (e.g. hospitals, clinics, general practice) versus emerging preventative services, increasingly delivered across a wider range of settings (e.g. workplaces, gyms, via apps or other digital tools) (Rickard et al. 2016).

In order to realise these benefits, however, consensus first needs to be reached as to which wellbeing questionnaire is most appropriate for monitoring health service impacts on wellbeing. Complicating this endeavour, however, is the fact that wellbeing research is, as a field, enormously varied. Currently, there is no single leading definition 
of the term "wellbeing", nor a widely agreed-upon and recommended approach to measuring this concept. Indeed, whilst numerous candidate wellbeing questionnaires have undergone validation for use in specific patient groups, their applicability to measuring general health service impact remains unclear. To date, a limited amount of research has explored service users' experiences interpreting and responding to different wellbeing questionnaires, and relatively few studies have attempted to delineate which factors are likely to facilitate the measurement process in this setting (Dolan et al. 2012; Linton et al. 2016; Mukuria et al. 2014).

Where research has been directed towards understanding the latter point, criticisms commonly levelled towards existing wellbeing questionnaires include the fact that they do not necessarily meaningfully reflect a patient's journey through a health service (Lee et al. 2013). For example, existing measures may ask respondents to reflect on their experience averaged over a preceding period (e.g. the previous 2 weeks) (Tennant et al. 2007; Topp et al. 2015). This approach makes it difficult to identify health-service specific drivers of wellbeing, aside from other potential broader causes. Moreover, wellbeing questionnaires that capture global-outcome states or subjective feelings leave healthcare providers with little insight into what exactly needs to change in order to improve the wellbeing of recipients (Batbaatar et al. 2017; Williams et al. 2016).

These points are reinforced in a comprehensive paper by Lee et al. (2013), in which authors argue that wellbeing questionnaires need to be deployed during pre-treatment, mid-treatment and post-treatment phases of care (Lee et al. 2013). Here, authors recommend that healthcare providers adopt a measure developed by Dolan et al. in a 2011 report produced by the UK Office for National Statistics (hereafter, referred to as "the ONS-4"measure)(Dolan et al. 2011). This measure taps evaluative, experiential and eudemonic aspects of wellbeing using four, 10point scales that rate overall life satisfaction, life worthwhileness and feelings of happiness and anxiety (Dolan et al. 2011).

Despite clear benefits of this tool, including brevity and the inclusion of a life satisfaction scale that can contribute data to economic evaluations, there remains almost one hundred alternative, validated questionnaires for possible use (Linton et al. 2016). It is evident, therefore, that more research is required to help healthcare providers who may be keen to measure wellbeing to choose which questionnaire is most appropriate for their own organisational context. To prove most useful, this work needs to take into account the views and needs of key stakeholders, including users of health and wellbeing services, as well as the healthcare staff responsible for overseeing the measurement process (Guise et al. 2013).

The aim of this study was fill this gap in the literature by exploring healthcare staff and service users' views on a range of wellbeing questionnaires for application in a healthcare setting. This research was conducted for Nuffield Health, the UK's largest not-for-profit healthcare organisation, at a time when the organisation was seeking to understand how best to measure wellbeing across their own diverse service portfolio. This includes treatment-related services such as private hospitals, diagnostic units, health clinics and primary care centres, corporate health services, physiotherapy, and cognitive behavioural therapy (CBT), as well as preventative health services such as a gym network and one-to-one fitness training.

For this study, a series of focus group were conducted with Nuffield Health staff and service users. Whilst we acknowledge that this research is limited in generalisability to 
recipients and providers of Nuffield Health services specifically, our findings are intended to help inform decision-making of other organisations also interested in measuring wellbeing. The primary research questions that this study aimed to answer were as follows:

- What do staff and service users understand by the term wellbeing, in general and in a healthcare context?

- What are staff and service users' views on a selection of candidate wellbeing questionnaires for possible adoption by Nuffield Health to monitor the impact of their health services?

- What are staff and service users' experiences with and recommendations for the process of measuring wellbeing across Nuffield Health services?

\section{Methods}

\section{Overview}

We employed a pragmatic research approach that retained rigour, yet aimed to address a specific practice need and was feasible for use in this real-world setting (Glasgow 2013). During January 2017, 10 stakeholder focus groups were carried out with NH staff and service users (total sample $N=64$ ). Eight of these focus groups included patients who had received care at a Nuffield Health hospital, as well as customers who had used Nuffield Health physiotherapy or gym services. One further group contained Nuffield Health Account Managers who are responsible for managing health and wellbeing services. One final group contained staff responsible for service delivery and service evaluation (e.g. one physiotherapist, two fitness managers, one deputy hospital matron, one physiologist responsible for conducting health assessments and a nutritional therapist). All focus groups comprised between five to seven persons. In addition, we also conducted three in-depth telephone interviews with members of Nuffield Health's Executive Board. Board members are ultimately responsible for directing the wellbeing agenda within the organisation and for deciding on which wellbeing measure to invest in.

\section{Recruitment}

Recruitment of staff and service users was conducted by an independent research agency (Flamingo: Global Insight \& Brand Consultancy, 2017). This agency is a member of the Medical Research Society and is accredited by the International Organisation for Standardization (see: BS ISO 20252:2012 for relevant standards; International Organization for Standardization, 2017). Agency staff were instructed to approach Nuffield Health service users registered on the organisation's research database, based on specifications to identify different "consumer segments" (see Table 1). Consumer segments were developed previously by Nuffield Health using factor analysis across a broader range of statements concerning attitudes to health that were subsequently clustered according to socio-demographic characteristics. The primary 
Table 1 Details of focus group participants and wellbeing tools review

\begin{tabular}{|c|c|c|}
\hline Group & Description of target consumer segment & Wellbeing tools reviewed \\
\hline \multicolumn{3}{|l|}{ Service Users } \\
\hline Group 1 & $\begin{array}{l}\text { "Able \& Stable" - } 65+\text { years old with income } \\
\text { slightly higher than UK average and } \\
\text { moderate interest in healthy lifestyle. }\end{array}$ & $\begin{array}{l}\text { World Health Organisation five } \\
\text { wellbeing index (WHO-5), } \\
\text { Warwick Edinburgh Mental } \\
\text { Wellbeing Scale } \\
\text { (WEMWBS) }\end{array}$ \\
\hline Group 2 & $\begin{array}{l}\text { "Comfy complacents" - 65+ years old with } \\
\text { income lower than UK average, with } \\
\text { lower interest in healthy lifestyle and } \\
\text { greater likelihood of ill health. }\end{array}$ & $\begin{array}{l}\text { Office for National Statistics } \\
\quad \text { Personal Wellbeing Tool } \\
\quad \text { (ONS-4), } \\
\text { Life satisfaction Questionnaire } \\
\text { (LISAT-9) }\end{array}$ \\
\hline Group 3 & $\begin{array}{l}\text { "Fit \& Focused" - } 35 \text { to } 34 \text { years old with } \\
\text { income above the UK average and high } \\
\text { motivation to look after their health. }\end{array}$ & $\begin{array}{l}\text { LISAT-9, } \\
\text { Patient Health Questionnaire } \\
\text { (PHQ-9) }\end{array}$ \\
\hline Group 4 & $\begin{array}{l}\text { "Happy Families" - } 45 \text { to } 54 \text { years old with } \\
\text { income levels at UK average, with average } \\
\text { interest in healthy lifestyle. }\end{array}$ & $\begin{array}{l}\text { ONS-4, } \\
\text { 36-item Short Form Survey } \\
\quad(\text { SF-36) }\end{array}$ \\
\hline Group 5 & $\begin{array}{l}\text { "Health Neglectors"- } 25 \text { to } 34 \text { years old with } \\
\text { income levels lower than UK average. } \\
\text { Healthy lifestyle is not a priority. }\end{array}$ & $\begin{array}{l}\text { Euroqol (EQ-5D-5 L), } \\
\text { Positive \& Negative Affect } \\
\quad \text { Schedule (PANAS) }\end{array}$ \\
\hline Group 6 & $\begin{array}{l}\text { "Healthy \& Wealthy" - 65+ years old with } \\
\text { income levels higher than UK average. } \\
\text { High motivation to look after their health. }\end{array}$ & $\begin{array}{l}\text { PHQ-9, } \\
\text { WEMWBS }\end{array}$ \\
\hline Group 7 & $\begin{array}{l}\text { "Hurried Healthies"- } 25 \text { to } 40 \text { years old with } \\
\text { income levels at UK average and moderate } \\
\text { interest in healthy lifestyles. }\end{array}$ & $\begin{array}{l}\text { WHO-5, } \\
\text { EQ-5D-5 L }\end{array}$ \\
\hline Group 8 & $\begin{array}{l}\text { "Sensible Seniors" - } 65+\text { years with low } \\
\text { income compared to the UK average, } \\
\text { higher likelihood of suffering from a } \\
\text { number of adverse health outcomes, and } \\
\text { have some interest in healthy lifestyle. }\end{array}$ & $\begin{array}{l}\text { PANAS, } \\
\text { SF-36 }\end{array}$ \\
\hline \multicolumn{3}{|l|}{ Staff } \\
\hline Group 9 & $\begin{array}{l}\text { Account Managers for fitness services, } \\
\text { marketing, clinical services, operational } \\
\text { support and physiology services. }\end{array}$ & All \\
\hline Group 10 & $\begin{array}{l}\text { Staff practitioners including fitness staff }(\times 2) \text {, } \\
\text { hospital matron, health and wellbeing } \\
\text { physiologist, physiotherapist, nutritional } \\
\text { therapist. }\end{array}$ & All \\
\hline
\end{tabular}

purpose of these segments is for the organisation to target marketing of products and services and explore differential patterns of service use.

Agency staff first approached all potential participants via phone and provided each with an overview of the aims of this study. Recruitment into staff focus groups was carried out internally. A convenience sample of Account Managers and healthcare practitioners were recruited from across the organisations' portfolio of services to ensure broad representation of different areas of work. 


\section{Procedure and Materials}

Focus groups were carried out by the research agency at three locations in the UK (Leeds, Birmingham and London) over a two-week period. All sessions were filmed and audiotaped with recorded verbal consent from members. Focus groups with patients and customers lasted approximately 1.5 hours each, and with staff members approximately 1 hour each. All focus groups followed the same format: prior to the session, participants were asked to carry out a pre-group task that was intended to facilitate subsequent group discussions (e.g. to reflect on own understandings of the term "wellbeing", and collate images that they felt reflected this understanding).

Topic guides were used to structure focus group discussions. These were designed to prompt general conversations surrounding the concept of "wellbeing" first, and then to allow group members to reflect on their own experiences of interacting with Nuffield Health services. Finally, service users were asked to review a selection of candidate wellbeing questionnaires, such that each questionnaire was filled out by at least two different groups, assigned in sequential order (see Table 1). Candidate measures included validated wellbeing questionnaires that Nuffield Health was already using across their portfolio, including in hospital-based research trials, for internal staff health and safety monitoring, during corporate health assessments and as part of existing wellbeing service provision for screening and diagnostic purposes. Focus group members were given time during sessions to read and fill out these questionnaires, before being asked to discuss their views on each.

Staff focus groups followed a similar structure, with members asked to reflect more generally on their understandings of the term "wellbeing", followed by a discussion of wellbeing in relation to their role at Nuffield Health. Further questions probed views on candidate wellbeing questionnaires, and experiences of managing service evaluation and measurement processes in the context of their own service provision. Table 2 provides a summary of the prompts that were used to direct group discussions.

The study protocol was reviewed and approved by Nuffield Health's Quality Committee and Research Expert Advisory Group to ensure that all procedures met relevant ethical and safety criteria. Details of the review process are provided in Supplementary Materials 1.

\section{Analysis}

Following group sessions, recordings were transcribed verbatim by an external transcription company (TakeNote Note Taking \& Transcription, UK, 2017). Authors SA and TLP read, reviewed and independently coded transcripts of group sessions. Both authors are trained to doctorate level in social science research methods, with experience analysing qualitative data in the context of applied research.

The analytical process involved identifying and clarifying relevant themes using thematic analysis (Braun and Clarke 2006). These two authors (SA and TLP) engaged in independent inductive coding of transcripts using ATLAS.ti software and Microsoft Excel, focussing on the content of discussions surrounding experiences measuring wellbeing for Nuffield Health, in addition to group members' reflections on the shortlisted wellbeing questionnaires. The final set of themes were decided upon and clarified through discussion between all authors. 
Table 2 Focus Group discussion prompts

\begin{tabular}{|c|c|}
\hline arget focus groups & cussion prompts \\
\hline $\begin{array}{l}\text { ervice Users (Groups } 1 \\
\text { to } 8 \text { ) }\end{array}$ & $\begin{array}{l}\text { Moderator will ask respondents to complete two questionnaires individually before } \\
\text { discussing as a group: } \\
\text { - What are your thoughts on what you just completed? } \\
\text { - Did what you filled in make sense in terms of your understanding of wellbeing? } \\
\text { - How do you feel having your wellbeing defined / determined by this type of question- } \\
\text { naire? } \\
\text { - Did this questionnaire address the elements of wellbeing that are important to you? } \\
\text { - How easy was it to fill in? } \\
\text { - Length? } \\
\text { - Language? } \\
\text { - Format? } \\
\text { - Would you prefer to fill this in on paper / online / both? } \\
\text { - Anything missing? Could be improved? }\end{array}$ \\
\hline Staff (Group 9 to 10 ) & $\begin{array}{l}\text { Moderator to ask staff to complete tools individually before discussing as a group. } \\
\text { Thinking about the content first of all } \\
\text { - What are your thoughts overall on what you just completed? } \\
\text { - Overall, does this make sense to NH and the service that you deliver? Why? Why not? } \\
\text { - Moderator to probe } \\
\text { - Did what you filled in make sense in terms of how wellbeing is measured? Does it feel } \\
\text { - What elements are working well here? Anything working less well? } \\
\text { - Did you feel that it addressed the key components of this concept? Anything in } \\
\text { particular? } \\
\text { - Did you feel like any key parts had been missed? Which? } \\
\text { - Hinking about the format and process of measurement } \\
\text { - Length? } \\
\text { - Language? } \\
\text { - Structure? } \\
\text { - How do you think your customers would feel about having to fill this in? } \\
\text { - What is working well? } \\
\text { - Anything working less well? Why? } \\
\text { - Anything missing? }\end{array}$ \\
\hline
\end{tabular}

\section{Results}

\section{Staff and Service User Understandings of the Term "Wellbeing"}

\section{Theme 1: Wellbeing as Balance}

In relation to our first research question, exploring understandings of the term "wellbeing", we were unable to identify a common definition or set of determinants of wellbeing that all focus group members endorsed; for example, some indicated that the elements required for their own wellbeing included social-life and work-life, while to others, physical health and lifestyle factors were paramount. Despite such diversity, however, a theme that consistently arose across all focus groups related to the idea that "balance" is integral to the concept of wellbeing. That is, focus group members considered it imperative that single areas of their lives did not demand too much attention, energy, time or resource, over and above other areas: 
"A good life balance, I think that's really important, you can always spend too much time on one thing or another." (Group 4)

"It's more important that I put everything in perspective in the whole of my life and not just do one or another, and for me that's, a balanced wellbeing is everything." (Group 5)

\section{Theme 2: Wellbeing as an Active Process}

In addition, when discussing their views on "wellbeing" in general, there was broad agreement across both staff and service user focus groups that this sense of balance must be actively maintained. Focus group members discussed how they strived to retain balance in the face of external challenges, such as major life events, like sickness or job changes, or in response to smaller, day-to-day hassles. Group members commonly shared instances of where they had felt tipped off balance and described strategies that they had adopted to regain equilibrium. Examples included changing their perspective on a situation, adapting their lifestyle, taking "time out" to relax and "re-charge", distracting themselves, socialising and seeking spiritual support:

“...so, I took a day off, I went away and I just sort of reassessed my situation and thought, I'm not going to accept any negativity from anyone ever again, if anyone's negative to me I'm going to turn it into a positive, I can't accept it, you know, 'cause it drags you down." (Group 8)

\section{Theme 3: Wellbeing in Relation to Treatment-Related Health Services}

When seeking care from a provider like Nuffield Health, one major theme that emerged was the importance of feeling supported and protected during a period of acute disruption and anxiety. For service users who had received a diagnosis or treatment in particular, the speed with which they were attended to was centrally important to their wellbeing, helping to ease anxiety and worry. Additionally, the competency of hospital staff was also an important factor in allaying worry and ensuring that patients and family members felt secure and safe while seeking care. Related to this, staff interpersonal skills were also seen as exerting an important, and often immediate, impact on wellbeing:

"You could be waiting for a diagnosis, or waiting to see someone for god knows how long, and that can put stress and worry on people." (Group 3)

"You're getting exposure to top professional people, doctors, whoever they might well be, and so that's very reassuring." (Group 2)

\section{Theme 4: Wellbeing in Relation to Preventative Health Services}

When asked to reflect on their experiences interacting with the types of preventative health services provided by Nuffield Health, group members were most familiar with 
fitness centres. Here, service users mentioned that the primary role that these centres played in their wellbeing was as a place they could go to take time out and relieve stress on a regular basis. For some, time alone in the gym was particularly valued as a means to help re-attain balance:

"If I've worked, if I have a bad day at work, I want to get out of the house, it's almost, if I want some time to myself, that's how I see it, going to the gym." (Group 7)

Health enhancing behaviour, like regular physical activity, was also mentioned by a number of focus group members as being a positive part of their normal routine, giving their day a pleasant structure or defined purpose, so promoting better wellbeing overall. Additionally, fitness centres themselves also appeared to play a key wellbeingpromoting role by acting as spaces that afford opportunities for positive and regular social interactions to occur.

Interestingly, conspicuous by their absence were comments relating to the physical effect of regularly accessing fitness centres, with very few group members mentioning that they use physical exercise as a means to enhance wellbeing by changing their physical appearance, altering their body in some way or to improve their fitness:

"I come here every day because this is my way of life, this is what I do." (Group 6)

"Morning routine for me, because when I retired I was just staying in bed and watching television, and I thought, 'I need to do something about this', so I joined, actually, the gym, so I go swimming most mornings, and that sets me up for the day." (Group 2)

\section{Staff and Service User Views on Candidate Wellbeing Questionnaires}

\section{Theme 5: Lack of Actionable Insight}

Considering our second research question pertaining to staff and service users views on a selection of candidate measurement tools for capturing wellbeing in a healthcare context, one clear theme to emerge was that questionnaires that provide a single outcome score that reflects a global end-state (e.g. WHO-5, WEMWBS), yield data that is too abstract and difficult to interpret in a meaningful way. For example, service users commented that these types of questionnaire are unable to pinpoint the independent influence of a health service, aside from other causal factors, on their own wellbeing. As such, the resultant data was not considered personally interesting, as it neither allow the respondent to gain insight into the causes of their own wellbeing, nor helped to direct their care:

"When you go into hospital for whatever reason, I think it's got to be a more personal approach, so I think filling out a form that says, um, I've been feeling 
cheerful and you have to give a score, I don't think that's the sort of question they should be asking you." (Group 1)

"What are they trying to gage from that, how I feel at the time? Or are they going to use that to give me different exercises to do at the gym? Or... so I can't see how they can take that on board." (Group 7)

\section{Theme 6: Screening Rather than Monitoring Tools}

Staff voiced similar concerns to service users regarding the purpose of wellbeing questionnaires in a healthcare context, suggesting that these may be more appropriate, and have a greater chance of being accepted, if they can provide actionable insight. Questionnaires that give a single global outcome score were, therefore, seen to offer scant guidance for staff on how to generate health service improvements. In general, staff members discussed how they saw the value of this type of wellbeing questionnaire lying in their potential use as screening tools. Staff focus groups frequently turned to discussions of how wellbeing questionnaires can help to identify individual patients with particularly low levels of wellbeing, so leading to further in-depth assessment that can then direct care or allow for a holistic treatment approach to be taken:

"Are we measuring something here, and then later? Or are we stratifying care? So are we using this tool and based on this outcome we will signpost. I think both are different" (Group 9)

"I think it would be a facilitator for joining up services. It would allow for conversation and recommendation to happen... it allows movement from maybe physio to Cognitive Behavioural Technology or other ones to link up and come back." (Group 9).

\section{Theme 7: Appropriateness}

A further theme to emerge in relation to wellbeing measurement in a healthcare context was the fact that the chosen questionnaire needs to accurately reflect patient understandings of wellbeing. Group members discussed how wellbeing questionnaires with a "mental" focus (e.g. detecting mental health disorders, or measuring affective states, such as the PANAS and PHQ-9) were seen as too "narrow" to reflect staff and service users broader understandings of wellbeing as balance. Group members also repeatedly noted that they construed wellbeing as incorporating a substantial "physical" component, which must also be captured by the chosen measurement tool. More specifically, the PHQ-9 was considered too "negative", whilst the PANAS was seen as limited in scope:

"I think this is just the wrong questionnaires, when mentioning wellbeing" (Group 6)

"It feels like it's only targeting your mental health and nothing about your physical health, so you need a mix of both." (Group 5) 
During focus groups, staff and selected service users were also asked to fill in and reflect upon health-related quality of life (HRQoL) measures, the EQ-5D-5 L and SF36. Nuffield Health and other healthcare organisations already routinely use these tools for the purposes of outcome monitoring, yet their relevance as potential measures of wellbeing is uncertain. Here, both staff and service users saw these questionnaires as more useful for patients suffering from debilitating health conditions or disabilities, and were not appropriate for use in currently healthy samples seeking preventative treatments, like exercise or stress management:

"I feel like the first part, is like, social services would ask if you like, your ability to do quite basic things, so I thought, "why am I answering questions about that'." (Group 5)

"This isn't relevant to me 'cause I'm physically, don't have problems if you know what I mean." (Group 7)

\section{Theme 8: Capturing a Fluctuating Phenomenon}

Two further wellbeing questionnaires that were reviewed in relation to our second research question incorporated indices of life satisfaction - the ONS-4, which also measures experiential and eudemonic wellbeing, and the LISAT-9, which captures degree of satisfaction with different life domains (e.g. leisure, work, finances etc.). For the ONS-4, service users commented favourably on the short time scale covered by this measure (e.g. happiness and anxiety in the last 24 hours). When considering these tools, and in particular the ONS-4, conversations tended to turn towards how wellbeing is construed as a constantly fluctuating phenomenon, varying considerably throughout the day, and so is hard to assign an average score to that reflects their experiences seeking treatment as a whole. As a result, service users viewed the ONS-4 as producing relatively unstable estimates of their wellbeing, especially if the measurement process is not repeated multiple times to obtain an averaged value:

"what about, 'how anxious did you feel yesterday', I mean, you can't say you weren't feeling anxious the whole day, might have felt anxious for five minutes, so where does that fall on your scale of 1 to 10." (Group 2)

"You can do that questionnaire 5 times a day and the score would be completely different." (Group 10)

\section{Theme 9: Isolating Cause and Effect}

Associated with theme 8, an additional theme to emerge concerned what causes fluctuations in wellbeing, both in general and in a health care context. Within discussions of this theme, the LISAT-9 was referred to favourably by service users as it has the benefit of pinpointing specific contributory causes of overall life satisfaction across a range of domain areas. Hence, this questionnaire was seen as valuable for promoting 
an understanding of cause-effect relationships. However, focus group members did add that this questionnaire incorporated domains that are not, and are unlikely to be, a target for wellbeing for a healthcare provider like Nuffield Health. For example, questions probing existing levels of satisfaction with sexual and financial situations were seen as intrusive and irrelevant when asked in this context. Further commentary centred on the phrasing of response scales, with the concept of "satisfaction" thought to be irrelevant to a number of questionnaire items:

"I would say personal circumstances, that's not really something that Nuffield Health has nothing to do it." (Group 3)

"I don't know why the doctor needs to know that my sexual situation is only rather satisfying at the moment" (Group 3)

\section{Staff and Service Users Experiences and Recommendations for Measuring Wellbeing in a Healthcare Context}

\section{Theme 10: Training and Support}

In terms of our third research question, exploring staff and service users' experiences with and recommendations for the process of measuring wellbeing across Nuffield Health services, one of the major themes to emerge from both staff focus groups and Executive Board Member interviews was a reticence to engage in widespread measurement across the organisation using mental health focussed questionnaires (e.g. PHQ-9, PANAS) without first receiving appropriate training and support. Staff discussed feeling uncertain that they were sufficiently knowledgeable to help service users identified as having distinct mental-health needs. As such, they suggested that relevant care pathways and infrastructure would first need to be developed before any routine measurement process is initiated:

"We would need to concentrate on is our duty of care to our employees to make sure that they are not burdened by what is identified. So the measure itself might be a light touch, but it might just be the catalyst that allows someone to say "well actually I regularly self harm" or "my husband beats me up"." (Executive Board 1)

"What are you going to do with the answer? Even if its signposting to somebody else... It would take a lot of training to get everyone to signpost." (Group 9)

\section{Theme 11: Highlighting Tangible Benefits}

In order to enhance uptake of wellbeing questionnaires in a health care context, both staff and service users commented on the fact that measurement must not only yield data that is useful for the organisation, but that can offer service users real and tangible benefits. This was either discussed in terms of assuring visible service improvements are made based on the data collected, that care is appropriately tailored, or that outcome 
data is fed-back to the user, with additional interpretation, for their own interest and to track changes and determinants of their own wellbeing over time:

"If we're relying on people to invest time and energy in it it's got to feel like it's giving them something back as a quid pro quo, on that basis, whatever that might be." (Executive board 2)

"If I'm filling out a questionnaire and I know what it was for, for my personal wellbeing, and stuff... I'm filling out something for me, personally" (Group 4)

\section{Theme 12: Confidentiality and Data Usage}

For many service users, the service context in which the wellbeing questionnaire is delivered appeared to be an important consideration and one that is likely to influence participation in the measurement process. Confidentiality concerns were frequently voiced, along with a sentiment that Nuffield Health may be over-reaching in asking wellbeing-related questions, especially in contexts outside of primary and secondary care. Associated with this were further conversations pertaining to the fact that clear explanations as to why the organisation wishes to collect this type of data are required before respondents to are likely to be willing to fill out any questionnaire:

"I think if you're filling this in, I'd be thinking to myself, you've got me filling this in, why do you want to know all of this." (group 6)

"I think it would have to be explained, because there's no causal link between that and going in for an operation, do you know what I mean, if somebody said, what does this have to do with that, it would need some explanation (Group 1)

\section{Theme 13: Practicalities and Burden}

Finally, in relation to our third research question, staff group members, in particular, discussed the practicalities involved in the measurement process. One point repeatedly alluded to was the fact that wellbeing measurement must not disrupt the flow of a consultation, nor replicate other questionnaires that are also used for routine monitoring, leading to potential redundancy in the outcome data collected:

"Trying to fit everyone's measurements into one thing would be very hard. As a physio you don't want them to repeat any of the questions you are going to ask them, as they will think, oh you've already asked me to fill that information in." (Group 10)

Commentary concerning measurement burden also frequently emerged, with staff members, as well as service users, suggesting that there is likely to be considerable unwillingness to spend time filling out wellbeing questionnaires, especially if no direct benefit to the service user is evident. This theme was also linked to further discussions 
surrounding mode of data collection, with service users clearly stating that they are unlikely to prioritise filling out any questionnaire outside the context of a direct consultation (e.g. if emailed subsequently), and tended to prefer an electronic medium rather than a paper-based approach:

\section{"If you try getting somebody to come in and fill in the gym they are already feeling time sensitive... they come into do a leisure activity, they are already wasting time... There is already a lot of paper work that they fill out and spend time doing” (Group 10)}

"If they tell you to go online and fill it in, or whatever, you have a million other things to do when you get home and you just can't be bothered." (Group 1)

\section{Discussion}

This stakeholder engagement exercise explored Nuffield Health staff and service user perspectives on candidate wellbeing questionnaires and summarises their views on the process of measuring wellbeing in both a preventative and treatment related health service setting. Our findings suggest that none of the candidate questionnaires reviewed by our participants were considered wholly suitable for their intended purposes. Common criticisms included irrelevance of question items and too great a focus on certain areas of wellbeing, to the expense of other relevant areas. Also frequently mentioned were the difficulties that respondents faced in interpreting numeric scales and the challenge of trying to assign a fixed "average" score to what is seen as a continually fluctuating and highly subjective phenomenon.

Additionally, our focus group members also provided commentary on the measurement process itself. Here, staff members discussed their concerns that wellbeing training and relevant care pathways must be implemented alongside measurement. This is to manage a scenario in which latent mental health conditions are revealed through questioning. Both staff and service users also highlighted the importance of ensuring data confidentiality, and that Nuffield Health must provide questionnaire recipients with a clear explanation of what they intend to use their data for prior to it's collection.

Considering our findings in the context of the existing literature, we could locate no other studies that have explored staff and service user perspectives on wellbeing questionnaires intended for monitoring service impact in a healthcare setting. Consulting broader qualitative research exploring alternative questionnaires used for a similar purpose (e.g. HRQoL questionnaires), however, issues akin to those revealed in our focus groups are highlighted. For example, like this study, previous research has also uncovered the importance of ensuring that the measurement process is embedded within a supportive service infrastructure, and that any insight gained is actually used to inform service improvements, rather than remaining solely a monitoring and data reporting exercise (Appleby and Delvin 2005; Kyte et al. 2013).

Moreover, again reflecting our findings, a recent study exploring service evaluation questionnaires (including the WEMWBS) used in the context of NHS-based psychological therapy found that service users suggested that these type of questionnaires may have some 
potential to elicit distress (Fornells-Ambrojo et al. 2017). In this study, respondents mentioned how such measurement was seen as potentially intrusive and upsetting for those who are seeking support for a mental health related issue. As such, care needs to be taken in choosing an appropriate wellbeing measure - one that is sensitive enough to identify patients with particularly low levels of wellbeing, but that does not elicit excess anxiety or distress. A similar finding is echoed in an earlier research study exploring nurses' views on HRQoL questionnaires employed for service monitoring purposes, in which interviewees also discussed the importance of using questionnaires that are considered appropriate for the context (Kyte et al. 2013).

In addition, similar to this study, existing research has also found that digital mediums are the preferred way to capture wellbeing data in healthcare settings. This channel is seen as particularly beneficial given that it allows data collection to occur outside of valuable consultation time (Coulter 2017). However, the possibility of using digital mediums to collect data at distance does raise the question of when, exactly, these measures should be deployed, by which specific mode (e.g. app, email, online interface) and how to encourage high response rates at distance. To the latter point, members of our focus groups expressed a clear preference for receiving feedback on their own wellbeing that is relevant to them specifically, often stating that they would be more willing to answer questionnaires if the personal benefit of doing so is made clear. Understanding individual cause-effect relationships in the context of their own wellbeing emerged as an area of interest to many of our participants. As such, any digital wellbeing measurement platform that is employed in a health service setting must be flexible enough to allow for a range of different possible questionnaires to be integrated and removed as appropriate. This permits data on wellbeing as an outcome variable to be captured, as well as also collecting information on a range of potential predictor variables of interest to the service provider as well as to the individual respondent. This approach, of including wellbeing questionnaires as just one tool in a wider battery of measures, is favoured by those leading research in this field (e.g. the Core Outcome Measures in Effectiveness Trials Initiative (http://www.comet-initiative. org), Patient-Reported Outcomes Measurement Information System (PROMIS) (http://www.healthmeasures.net), as well as by providers of novel digital wellbeing measurement solutions for patient groups (e.g. Umotif (http://www.umotif.com)).

One further key finding to arise from our focus groups was that both staff and service users appeared to understand the term "wellbeing" as encompassing the notion of "balance". As such, one useful next step for Nuffield Health may be to identify wellbeing questionnaires that capture this emergent definition more closely. Considering research that has already explored definitions of wellbeing, we note that this concept of balance aligns to an pre-existing theory variously described as "Dynamic Equilibrium Theory", "Set Point Theory" or the "Hedonic Treadmill"(Diener et al. 2009; Headey 2006). Here, wellbeing is described as an adaptive process, with this theory proposing that individuals readily habituate to changing life circumstances, both positive and negative, so leading to relatively stable wellbeing over time (Diener et al. 2009). Questionnaires used to tap wellbeing according to these theories include mood scales, personality inventories and evaluative questionnaires, combined together into a single questionnaire bank (Tomyn and Cummins 2011). Therefore, a potentially 
valuable next step for Nuffield Health would be to conduct further research pilot testing these extended questionnaires in practice, in order to understand their relevance as common outcome measures across their portfolio of different services.

\section{Strengths and Limitations of this Study}

This study addresses a clear gap within the existing evidence-base by providing insight into stakeholder perspectives on candidate wellbeing questionnaires in a non-traditional health service setting, using a pragmatic approach that ensures findings retain real practical relevance. Indeed, as one recent publication shows, only around $10 \%$ of studies that have explored approaches to health service evaluation have actually asked patients which outcomes they consider worth measuring (Wiering et al. 2017). Given that comprehensive monitoring of service impact requires that patients willingly contribute their data to the measurement process, this omission has the potential to result in service evaluation protocols that do not engage patients as intended, so producing insufficient or potentially biased data on which to base subsequent decisionmaking.

We do, however, acknowledge that Nuffield Health is a unique healthcare provider, offering a range of services that differ to those delivered by others, including the UK NHS. In particular, Nuffield Health provides services at direct cost to the patient, and covers those delivered outside of traditional primary or secondary care settings. As such, the views of staff and service users represented in this study may not reflect those of their counterparts receiving and delivering care through publically funded health services, where expectations, opportunities and experiences of treatment may differ.

\section{Conclusion}

Wellbeing is complex and subjective concept, and existing questionnaires used by Nuffield Health to monitor the impact of their service provision on broader patient wellbeing may not well reflect the experiences of their staff and service users. In a health setting, wellbeing questionnaires need to yield actionable insight that proves useful for both the individual and the organisation and should tap a concept that is readily understood by respondents. Stakeholder engagement using focus groups is a pragmatic approach that can inform the wellbeing measurement agenda by exploring the viewpoints of those affected by this process and is recommended as a useful way to understand wellbeing measurement-related issues in this setting.

Acknowledgements The authors thank Flamingo staff Milly Fearon and Yasmin Scott and who recruited and ran focus group sessions with staff and service users.

Funding This research was funded by Nuffield Health. Authors SA, RO, CK and BK were all NH employees at the time of writing. 


\title{
Compliance with Ethical Standards
}

\author{
Disclaimers None
}

Open Access This article is distributed under the terms of the Creative Commons Attribution 4.0 International License (http://creativecommons.org/licenses/by/4.0/), which permits unrestricted use, distribution, and reproduction in any medium, provided you give appropriate credit to the original author(s) and the source, provide a link to the Creative Commons license, and indicate if changes were made.

\section{References}

Appleby, J., \& Delvin, N. (2005). Measuring NHS success. Can patients' views on health outcomes help to manage performance? London: King's Fund Publications.

Batbaatar, E., Dorjdagva, J., Luvsannyam, A., Savino, M. M., \& Amenta, P. (2017). Determinants of patient satisfaction: a systematic review. Perspectives in Public Health, 137(2), 89-101. https://doi.org/10.1177 /1757913916634136.

Benning, T. M., Alayli-Goebbels, A. F. G., Aarts, M.-J., Stolk, E., de Wit, G. A., Prenger, R., ... Evers, S. M. A. A. (2015). Exploring outcomes to consider in economic evaluations of health promotion programs: what broader non-health outcomes matter most? BMC Health Services Research, 15(1), 266. https://doi. org/10.1186/s12913-015-0908-y.

Braun, V., \& Clarke, V. (2006). Using thematic analysis in psychology. Qualitative Research in Psychology, 3(2), 77-101. https://doi.org/10.1191/1478088706qp063oa.

Core Outcome Measures in Effectiveness Trials Initiative. (2017). Retrieved December 2 2017, from http://www.comet-initiative.org.

Coulter, A. (2017). Measuring what matters to patients. BMJ, j816. https://doi.org/10.1136/bmj.j816.

Diener, E., Wirtz, D., Tov, W., Kim-Prieto, C., Choi, D., Oishi, S., \& Biswas-Diener, R. (2009). New wellbeing measures: short scales to assess flourishing and positive and negative feelings. Social Indicators Research, 97(2), 143-156. https://doi.org/10.1007/s11205-009-9493-y.

Dolan, P., Layard, R., \& Metcalfe, R. (2011). Measuring subjective wellbeing for public policy. Office for National Statistics, 6(February), 19-215. https://doi.org/10.2752/174589311X12961584845846.

Dolan, P., Lee, H., \& Peasgood, T. (2012). Losing sight of the wood for the trees: Some issues in describing and valuing health, and another possible approach. PharmacoEconomics, 30(11), 1035-1049. https://doi. org/10.2165/11593040-000000000-00000.

Dooris, M., Farrier, A., \& Froggett, L. (2017). Wellbeing: the challenge of "operationalising" an holistic concept within a reductionist public health programme. Perspectives in Public Health, 1757913917711204, 93-99. https://doi.org/10.1177/1757913917711204.

Enns, J., Holmqvist, M., Wener, P., Halas, G., Rothney, J., Schultz, A., et al. (2016). Mapping interventions that promote mental health in the general population: a scoping review of reviews. Preventive Medicine, 87, 70-80. https://doi.org/10.1016/j.ypmed.2016.02.022.

Ettorchi-Tardy, A., Levif, M., \& Michel, P. (2012). Benchmarking: a method for continuous quality improvement in health. Healthcare Policy Politiques de Santé, 7(4), e101-e119. https://doi.org/10.12927 /hcpol.2012.22872.

Flamingo: Global Insight \& Brand Consultancy. (n.d.). Retrieved August 7, 2017, from http://flamingogroup. com

Fornells-Ambrojo, M., Johns, L., Onwumere, J., Garety, P., Milosh, C., Iredale, C., Peters, E., Webster, A., \& Jolley, S. (2017). Experience of outcome monitoring in service users with psychosis: findings from an improving access to psychological therapies for people with severe mental illness (IAPT-SMI) demonstration site. British Journal of Clinical Psychology, 56(3), 253-272. https://doi.org/10.1111/bjc.12136.

Glasgow, R. E. (2013). What does it mean to be pragmatic? Pragmatic methods, measures, and models to facilitate research translation. Health Education and Behavior, 40(3), 257-265. https://doi.org/10.1177 $/ 1090198113486805$.

Guise, J.-M., O’Haire, C., McPheeters, M., Most, C., LaBrant, L., Lee, K., et al. (2013). A practice-based tool for engaging stakeholders in future research: a synthesis of current practices. Journal of Clinical Epidemiology, 66(6), 666-674. https://doi.org/10.1016/j.jclinepi.2012.12.010. 
Headey, B. (2006). Subjective well-being: revisions to dynamic equilibrium theory using national panel data and panel regression methods. Social Indicators Research, 79(3), 369-403. https://doi.org/10.1007 /s11205-005-5381-2.

Hilton, L., Hempel, S., Ewing, B. A., Apaydin, E., Xenakis, L., Newberry, S., Colaiaco, B., Maher, A. R., Shanman, R. M., Sorbero, M. E., \& Maglione, M. A. (2017). Mindfulness meditation for chronic pain: systematic review and meta-analysis. Annals of Behavioral Medicine, 51(2), 199-213. https://doi.org/10.1007 /s12160-016-9844-2.

ISO. (n.d.). International Organization for Standardization. Retrieved August 7, 2017, from https://www.iso. org/home.html.

Kyte, D., Ives, J., Draper, H., Keeley, T., \& Calvert, M. (2013). Inconsistencies in quality of life data collection in clinical trials: a potential source of bias? Interviews with research nurses and trialists. PLoS One, 4, 8(10):e76625. https://doi.org/10.1371/journal.pone.0076625.

Lee, H., Vlaev, I., King, D., Mayer, E., Darzi, A., \& Dolan, P. (2013). Subjective well-being and the measurement of quality in healthcare. Social Science \& Medicine, 99, 27-34. https://doi.org/10.1016/j. socscimed.2013.09.027.

Linton, M.-J., Dieppe, P., \& Medina-Lara, A. (2016). Review of 99 self-report measures for assessing wellbeing in adults: exploring dimensions of well-being and developments over time. BMJ Open, 6(7), e010641. https://doi.org/10.1136/bmjopen-2015-010641.

Mikolasek, M., Berg, J., Witt, C. M., \& Barth, J. (2017). Effectiveness of mindfulness- and relaxation-based eHealth interventions for patients with medical conditions: A systematic review and synthesis. International Journal of Behavioral Medicine, 25, 1-16. https://doi.org/10.1007/s12529-017-9679-7.

Mukuria, C., Rowen, D., Peasgood, T., \& Brazier, J. (2014). An empirical comparison of well-being measures used in UK.

Murray, M., Murray, L., \& Donnelly, M. (2016). Systematic review of interventions to improve the psychological well-being of general practitioners. BMC Family Practice, 17(1), 36. https://doi.org/10.1186 /s12875-016-0431-1.

Naylor, C., Imison, C., Addicott, R., Buck, D., Goodwin, N., Harrison, T., ... Curry, N. (2015). Transforming our health care system: Ten priorities for commissioners.

NHS. (2014). NHS Five Year Forward View.

NHS England. (2018). The improving access to psychological therapies (IAPT) pathway for people with longterm physical health conditions and medically unexplained symptoms.

Nuffield Health. (2018). Retrieved May 14 2018, From https:/www.nuffieldhealth.com/article/innovation.

Patient-Reported Outcomes Measurement Information System (PROMIS). (2018). Retrieved December 2 2018, From http://www.healthmeasures.net.

Rickard, N., Arjmand, H.-A., Bakker, D., \& Seabrook, E. (2016). Development of a Mobile phone app to support self-monitoring of emotional well-being: a mental health digital innovation. JMIR Mental Health, 3(4), e49. https://doi.org/10.2196/mental.6202.

TakeNote Note Taking \& Transcription. (n.d.). Retrieved August 7, 2017, from https://takenotetyping.com/.

Tennant, R., Hiller, L., Fishwick, R., Platt, S., Joseph, S., Weich, S., ... Stewart-Brown, S. (2007). The Warwick-Edinburgh mental well-being scale (WEMWBS): development and UK validation. Health and Quality of Life Outcomes, 5(1), 63. https://doi.org/10.1186/1477-7525-5-63.

Tomyn, A. J., \& Cummins, R. A. (2011). Subjective wellbeing and homeostatically protected mood: theory validation with adolescents. Journal of Happiness Studies, 12(5), 897-914. https://doi.org/10.1007 /s10902-010-9235-5.

Topp, C. W., Østergaard, S. D., Søndergaard, S., \& Bech, P. (2015). The WHO-5 well-being index: a systematic review of the literature. Psychotherapy and Psychosomatics, 84(3), 167-176. https://doi. org/10.1159/000376585.

Wiering, B., de Boer, D., \& Delnoij, D. (2017). Patient involvement in the development of patient reported outcome measures: a scoping review. Health Expectations: An International Journal of Public Participation in Health Care and Health Policy, 20(1), 11-23.

Williams, V., Kinnear, D., \& Victor, C. (2016). 'It's the little things that count': healthcare professionals' views on delivering dignified care: a qualitative study. Journal of Advanced Nursing, 72(4), 782-790. https://doi.org/10.1111/jan.12878. 


\section{Affiliations}

Sophie Attwood ${ }^{1} \cdot$ Tarra L. Penney $^{1} \cdot$ Rachael O'Leary $^{1} \cdot$ Charlotte Klahn $^{1}$ • Benjamin Kelly ${ }^{1}$

Tarra L. Penney

Tarra@pandacare.ca

Rachael O'Leary

Rachael.oleary@nuffieldhealth.com

Charlotte Klahn

charlotte.klahn@nuffieldhealth.com

Benjamin Kelly

Benjamin.kelly@nuffieldhealth.com

1 Nuffield Health, London, UK 